Original Article

\title{
Measurement the thickness of the transverse abdominal muscle in different tasks
}

\author{
Ling Pang, Ns ${ }^{1)}$, Liquan Yin ${ }^{1)}$, Kimiko Tajiri, MS, Ns ${ }^{2)}$, Ming Huo, PT, $\mathrm{PhD}^{3)^{*}}$, \\ Hitoshi Maruyama, PT, $\mathrm{PhD}^{4)}$ \\ 1) China-Japan Friendship Hospital Affiliated Jilin University, China \\ 2) Bukkyo University, Japan \\ 3) Himeji Dokkyo University: 7-2-1 Kami-ono, Himeji City, Hyogo 670-8524, Japan \\ 4) International University of Health and Welfare, Japan
}

\begin{abstract}
Purpose] This study examined the measurement of the thickness of the transverse abdominal muscle in different tasks. [Subjects and Methods] The subjects were eleven healthy adult females. Thicknesses of transverse abdominal muscle were measured in seven tasks in the supine position. The tasks were: 1) Resting state, 2) Maximal contraction of transverse abdominal muscle, 3) Maximal contraction of levator ani muscle, 4) Maximal simultaneous contraction of both transverse abdominal muscle and levator ani muscle, 5) Maximal simultaneous contraction of both transverse abdominal muscle and levator ani muscle with front side resistance added to both knee, 6) Maximal simultaneous contraction of both transverse abdominal muscle and levator ani muscle with diagonal resistance added to both knees, and 7) Maximal simultaneous contraction of both transverse abdominal muscle and levator ani muscle with lateral resistance added to both knees. [Results] The thicknesses of transverse abdominal muscle during maximal simultaneous contraction and maximal simultaneous contraction with resistance were greater than during the resting state. [Conclusion] The muscle output during simultaneous contraction and resistance movement were larger than that of each individual muscle.

Key words: Transverse abdominal muscle, Exercise, Levator ani muscle
\end{abstract}

(This article was submitted Sep. 10, 2016, and was accepted Oct. 27, 2016)

\section{INTRODUCTION}

Urinary incontinence (UI) is well-known to profoundly affect the QOL (quality of life) of women. Pelvic floor muscle exercise (PFM) has been reported to be from $50 \%$ to $69 \%$ effective at reducing urine loss episodes in women ${ }^{1-4)}$. In our previous study, we found a significant relationship between the thickness of the transverse abdominal muscle (TA) and the iEMG of the levator ani muscle (LA). This result suggests that changes in the thickness of the TA may be used to indicate changes in the electrical activity of the PFM. In our other previous study, the TA and LA co-contraction exercise intervention increases the thickness of the TA and may be recommended to improve SUI in middle-aged women ${ }^{5}$.

The purpose of this study was to devise a new urinary incontinence exercise using co-contraction of both the transverse abdominal muscle and pelvic floor muscle with resistance, and examined the measurement of the thickness of the transverse abdominal muscle (TA) in different tasks in middle-aged women.

*Corresponding author. Ming Huo (E-mail: huoming8@gmail.com)

(C2017 The Society of Physical Therapy Science. Published by IPEC Inc.

This is an open-access article distributed under the terms of the Creative Commons Attribution Non-Commercial No Derivatives (by-nc-nd) License $<$ http://creativecommons.org/licenses/by-nc-nd/4.0/>. 


\section{SUBJECTS AND METHODS}

The subjects were eleven healthy adult women: age, $52.9 \pm 8.0 \mathrm{yrs}$ (mean \pm standard deviation); height, $159.3 \pm 5.7 \mathrm{~cm}$; weight, $58.9 \pm 9.8 \mathrm{~kg}$. All subjects gave their informed consent to participate in this study. All experimental procedures in this study were reviewed and approved by the Ethical Review Committee of Jilin Dianli Hospital (JLDL2015-018).

The thickness of the TA was measured using ultrasound. The thickness of the TA was measured in all subjects under seven conditions at random in the supine position. 1) The first condition was the resting state. 2) The second condition was maximal contraction of the TA. For this, the subjects were instructed to draw in the lower abdominal wall toward the spine, an action that specifically activates the TA. The subjects were asked to breathe in a relaxed manner. No movement of the lumbar spine was allowed. 3) The third condition was maximal contraction of the LA. For this the subjects were instructed to contract the muscles around the vagina "like a drawstring" and to lift them internally. No posterior tilt of the pelvis was allowed. There was no instruction to either use or not use the abdominal muscles. 4) The fourth condition was maximal co-contraction of both the TA and LA. Subjects were instructed to draw in the lower abdominal wall toward the spine, an action that specifically activates the TA. When the TA sustained isometric contraction, the subjects were instructed to contract the muscles around the vagina "like a drawstring" to lift them internally and to keep this position for 5 seconds. 5) Maximal simultaneous contraction of both TA and LA with front side resistance added to both knee, 6) Maximal simultaneous contraction of both TA and LA with diagonal resistance added to both knees, and 7) Maximal simultaneous contraction of both TA and LA with lateral resistance added to both knees.

Under each condition, the subjects were in the supine position with the knees flexed at $90^{\circ}$ and a pillow under the head. A Biofeedback Stabilizer was used to provide visual feedback. The three-chamber pressure cells were placed under the lumbar spine, and the subjects were asked to keep the baseline at $40 \mathrm{mmHg}$. If the pressure of the Biofeedback Stabilizer decreased under conditions $2-7$, abdominal muscle re-education was provided by a physical therapist.

Ultrasound images of the anterolateral abdominal wall were obtained using a ultrasound (SonoSite 180PLUS, B mode, $5 \mathrm{MHz}$ linear transducer). Gel was interposed between the transducer and the skin. The transducer was positioned adjacent to and perpendicular to the abdominal wall, $25 \mathrm{~mm}$ anteromedial to the midpoint between the ribs and ilium on the midaxillary line, and parallel to the muscle fibers of the transversus abdominis ${ }^{6)}$. The same person, a midwife, made the measurements to avoid inter-rater errors. All thickness measurements were of muscle only, that is, between the fascia boundaries.

The resistance force during maximal simultaneous contraction of TA and LA was measured by two hand-held dynamometers (ANIMA uTas MT1, HHD) held in both hands of the measurer, using the tester function of HHD. A resistance force was added to both knees of the subjects. The resistance force was the maximum resistance that didn't appear as for the trunk shake of the subject. The mean value of the maximum resistance force of the right and left hands was assumed to be a representative value.

One-way analyses of variance and multiple comparisons (Bonferroni test) were used to test for statistically significant differences, and the factor was thickness of TA. Data were analyzed using SPSS Ver. 17.0 for Windows. The chosen level of statistical significance was 0.05 .

\section{RESULTS}

The thicknesses of TA during maximal simultaneous contraction and maximal simultaneous contraction with resistance were greater than during the resting state (Table 1).

The resistance force during maximal simultaneous contraction with front side resistance added to both knee was $7.90 \pm$ $2.5 \mathrm{~kg}, 7.83 \pm 1.6 \mathrm{~kg}$ with diagonal resistance added to both knees, and $7.52 \pm 1.8 \mathrm{~kg}$ with lateral resistance added to both knees with no significant difference.

Table 1. Effect of thickness of TA for each task

\begin{tabular}{lll}
\hline Task & \multicolumn{2}{c}{ Thicknesses of TA $(\mathrm{mm})$} \\
\hline Resting state $^{\mathrm{a}}$ & $2.5 \pm 0.5$ & $\mathrm{~d}>\mathrm{a}^{*}$ \\
Maximal contraction of TA $^{\mathrm{b}}$ & $3.8 \pm 1.4$ & $\mathrm{e}^{*} \mathrm{a}^{*}$ \\
Maximal contraction of LA $^{\mathrm{c}}$ & $3.5 \pm 1.1$ & ${\mathrm{f}>\mathrm{a}^{* *}, \mathrm{c}^{*}}^{*}$ \\
Maximal simultaneous contraction of both TA and LA $^{\mathrm{d}}$ & $3.9 \pm 1.3$ & $\mathrm{~g}^{*} \mathrm{a}^{* *}, \mathrm{~b}^{*}, \mathrm{c}^{*}$ \\
Maximal simultaneous contraction of both TA and LA with front side resistance $^{\mathrm{e}}$ & $4.0 \pm 1.2$ & \\
Maximal simultaneous contraction of both TA and LA with diagonal resistance $^{\mathrm{f}}$ & $4.3 \pm 1.3$ \\
Maximal simultaneous contraction of both TA and LA with lateral resistance $^{\mathrm{g}}$ & $4.8 \pm 1.6$ \\
\hline
\end{tabular}

Mean \pm SD. $* \mathrm{p}<0.05, * * \mathrm{p}<0.01$

TA: transverse abdominal muscle. LA: levator ani muscle 


\section{DISCUSSION}

The thicknesses of TA during maximal simultaneous contraction and maximal simultaneous contraction with resistance were greater than during the resting state. The maximal simultaneous contraction of both TA and LA with lateral resistance was greater than the maximal contraction of TA and the maximal contraction of LA. The muscle output during simultaneous contraction and simultaneous contraction with resistance were larger than that of each individual muscle not only for TA but also for LA.

In clinic intervention training of TA, the simultaneous contraction with resistance exercise increases the thickness of the TA and may be recommended to improve UI in middle-aged women. The simultaneous contraction of both TA and LA may be recommended to apply as a home exercise.

Further investigations will need to weigh the training effect for women with urine incontinence in a prospective intervention study.

\section{REFERENCES}

1) Henalla SM, Hutchins CJ, Robinson P, et al.: Non-operative methods in the treatment of female genuine stress incontinence of urine. J Ob Gyn, 1989, 9: 222-225. [CrossRef]

2) Goode PS, Burgio KL, Locher JL, et al.: Effect of behavioral training with or without pelvic floor electrical stimulation on stress incontinence in women: a randomized controlled trial. JAMA, 2003, 290: 345-352. [Medline] [CrossRef]

3) Hay-Smith EJ, Dumoulin C: Pelvic floor muscle training versus no treatment, or inactive control treatments, for urinary incontinence in women. Cochrane Database Syst Rev, 2006, (1): CD005654. [Medline]

4) Borello-France DF, Zyczynski HM, Downey PA, et al.: Effect of pelvic-floor muscle exercise position on continence and quality-of-life outcomes in women with stress urinary incontinence. Phys Ther, 2006, 86: 974-986. [Medline]

5) Tajiri K, Huo M, Maruyama H: Effects of co-contraction of both transverse abdominal muscle and pelvic floor muscle exercises for stress urinary incontinence: a randomized controlled trial. J Phys Ther Sci, 2014, 26: 1161-1163. [Medline] [CrossRef]

6) Critchley D: Instructing pelvic floor contraction facilitates transversus abdominis thickness increase during low-abdominal hollowing. Physiother Res Int, 2002, 7: 65-75. [Medline] [CrossRef] 